\title{
Factors Contributing to Inconsistent Use of Family Planning Services at St Joseph's Hospital Catchment Area
}

\author{
Article by Palesa Monamane \\ Public Health, Texila American University \\ E-mail: Palesamonamane@gmail.com
}

\begin{abstract}
Family planning is referred to as controlling the number of children a couple wishes to have, interval between births by means of artificial contraception or voluntary surgical contraception. It involves the use of contraceptives, other techniques which include sexual health education, prevention, and management of sexually transmitted diseases, pre-conception counselling and infertility management (WHO:2010)

Family planning services are comprehensive and enable an individual to have an informed decision regarding available methods. It considers maternal health as motherhood has risks especially early and late motherhood and has been seen as having benefits for the well- being of women, community and society at large.

Despite the benefits and modern methods that are used women still do not come for family planning services at recommended time. That is, there is still inconsistent use of family planning services by the clientele.
\end{abstract}

\section{Background}

St Joseph's Hospital is a catholic institution and has a maternity unit where majority of women come to deliver. It is situated in the Southern region of the main town called Maseru, and about $35 \mathrm{~km}$ from town. Prior and after delivery women are given health education on family planning services offered. This institution does not offer family planning services due to its religious belief but women are referred to the clinics around St Joseph's catchment area for family planning services.

Majority of family planning services are provided by the government integrating services with existing maternal and child health services and are provided freely throughout the country. Awareness in family planning methods is high among women as they can reveal one method and how to use that method. The services are provided throughout the week catering even for working women between ages 18-44. Health education is provided on the methods available so that a woman can select the method with full information.

Family planning is known to improve maternal health but inconsistent use makes it difficult to achieve especially in developing countries like Lesotho. In sub Saharan countries uncontrolled population growth has led to hindrance in attainment of development and health goals. Though it has been broadened to reach the most remote areas and it is supported by donors such as United Nations Fund for Population Activities (UNFPA) as well as to monitor the use of family planning services. It has also broadened its focus on addressing two reproductive and health rights being the rights of women to be informed and have access to safe, effective, affordable and acceptable methods of family planning of their choice. The choice of methods used for regulation of fertility should not be against the law. (The nation:2018)

Although much work has been done to increase access, countries have made significant progress such as Latin America in increasing contraceptive security thereby decreasing total fertility rate and through the help of the donors such as United States Agency for international Development (USAID). (deliver.jsi.com)

The regional initiative brings countries together to share lessons learned and solutions to consistent use into national results.

$26^{\text {th }}$ September, 2016 was dedicated as the World Contraception Day whereby progress has been made with regard to use of family planning services steered by international initiatives promoting access to sexual and reproductive health. Despite all these there still large differences between 
countries as over 200 million women would like to avoid pregnancy but still need to use methods effectively(who.int/reproductivehealth).

\section{Problem statement}

Family planning services have been in use for a longer time with the aim of improving maternal and child health, maternal mortality is still high in the rural areas an prevalence of HIV remains at $24.6 \%$, but inconsistent use of services is escalating. At St Joseph's catchment area about 181 women are seen who were in need of family planning services in September, 2016. The expectation was to see about 200 women and necessary services such as counselling on the use of a method, other options, health education and when to return for follow up are provided but despite all these, some inconsistencies on the use have been observed. According to the statistics 19 women did not show up in that month and that is about $9.5 \%$ of women missed. In October 2016, 154 women were seen and commodities were issued to them. The expectation was also to see 200 women, so fourty six women were not seen. A total of $23 \%$ was not seen for family planning services despite the time for follow up stated. It has also been observed in most African countries that they have lower rates of contraceptive use with highest mortality rates, infant and child mortality (Regional variations June 2016).

These have been observed on women aged 20-35years of age. So, the researcher wants to find out factors that contribute to inconsistent use of family planning services.

\section{Purpose}

To investigate the contributing factors to inconsistent use of family planning services.

\section{Objectives}

To identify the contributing factors to inconsistent use of family planning services.

To recommend acceptable strategies that can be applied to increase family planning services use.

\section{Literature review}

This entails contributions of various authors in order to come up with relevant literature regarding inconsistent use of family planning services whereby two groups of women were interviewed in one hospital in order to determine consistency on the use of family planning services. Majority relied on misinformation and likely to make errors in estimating the risk of pregnancy.

Family planning services are used by majority of women in the child-bearing age. They use the services as they have got plans and would like to achieve those in order to reach their set goals. A variety of methods are used which provide a woman a choice of a method. Despite the variation in methods some take control over contraception in their relationship and rarely deviate from their plans while other group of women reported that they do not want to have babies at their age but do not consistently use family planning services. Of all the women who reported not to desire to be pregnant $17 \%$ reported unplanned pregnancies due to inconsistent use and were not happy with the outcome. (who int reproductive health-family planning 2016).

Age of a client plays a role as clients believe that at a certain age one cannot fall pregnant even if she uses contraceptives inconsistently and others believe that if they engage in unprotected sex without getting pregnant it means they are infertile. One of the clients was asked whether she had engaged in an unprotected sex and reported that she did not consistently use a condom as she thought she would not get pregnant. Other women have made conclusions that they are infertile as they could not become pregnant and this influenced their inconsistency in the use of family planning services. (Consistent and inconsistent contraception among women 20-29. 2011).

Pregnancies among contraceptive users account for nearly half of all unintended pregnancies and are due to inconsistent use, therefore a study was undertaken nationally to a representative sample of 1,978 women aged 18-44 using contraceptives to examine factors associated with inconsistent use. Results revealed that $18 \%$ were using hormonal methods, $7 \%$ long acting injectables, 5\% IUD, 32\% male condoms, and $12 \%$ other methods while $6 \%$ periodic abstinence. $38 \%$ of women reported using their current method mostly because they disliked other options, while some $58 \%$ of women were using were using condoms because they did not like other methods. Women who were not completely 
satisfied with their method were more likely than others to use the method inconsistently, especially pill and condom users. (Darroch and Frost:2008).

Other factors that influences consistency in use of family planning services are attitudes of male partners, they can negatively influence consistency with their own behaviour and this can lead to inconsistencies in use. While others can influence positively in different ways by even reminding the partner on the use of services. Frequently reported side effects encourage a woman to quit using the services without notifying the service provider, though switching of the method can be effective but women prefer to use a new method and be effective within a very short time.

Some women have self efficacy with regard to the use family planning services as they see the benefits of using services while others have a low efficacy and end up being inconsistent on the use of family planning services. Health belief model expectation is that of perceived self efficacy to perform an action based on motivation. When there is lack of firm motivation family planning service use become inconsistent. The most recent survey of family growth found that $69 \%$ of pregnancies among Black women and $54 \%$ of Hispanics were unintended compared to $40 \%$ among White women and one of the reasons is inconsistent use of family planning services (Disparities in Family planning Outcomes. 2001).

Inconsistencies in use is also associated with low educational attainment, low income demography, greater number of sexual partners, access issues, mental status-stress.

Some women reported limited access transportation in order to access the clinics. Efforts are being done to increase access as Lesotho Planned Parenthood Association with the support of UNFPA has selected some days to reach remote areas in order to provide family planning commodities and also to meet the unmet need of family planning.

It has been observed that women come for family planning services without the knowledge of the spouse as spouses are against the use. Therefore, when their spouses are around or at home, they do not come for services due to fear that they will be forbidden to come for services, this associated with behavioural sabotage as they are not allowed to use the services. About 8,6\% of women in United States have reported that partners did not want them to use family planning commodities. (Wikipedia.org/reproductive control sabotage).

Due to the findings related to inconsistent use of family planning services, family planning plays a major part in controlling reproduction and needs to be reviewed as well as identifying how it could be expanded to address problems that are brought about by inconsistent use.

\section{Methodology}

\section{Introduction}

Entails how the study was carried out, research design, target population, sample size, and data collection.

\section{Research design}

A simple descriptive design was used, with the aim of searching for accurate information regarding factors contributing to inconsistent use of family planning services.

\section{Target population}

All women who attended family planning services at St Joseph's catchment area.

\section{Sample size}

Sample was 20 women

\section{Sampling method}

Non-probability convenience sampling method was used as those who were selected were readily available. They were given information about the study and those who wished to take part were identified. 


\section{Instrument}

A semi structured interview guide was used as it consisted of open and closed ended questions, with open ended questions allowing them to respond as they come to identifying factors contributing to inconsistent use of family planning services closed ended facilitated coding and analysis of data.

\section{Pilot study}

A mini dummy run was done with five women in one of the clinics around St Joseph's catchment area in order to assess the feasibility of the study. This was done in order to assist in making adjustments on the actual project.

\section{Data collection}

Data was collected every Monday for four weeks as the women come for family planning services every Monday. After identifying participants information was given so as for them to give consent voluntarily. Interview guide was issued to them to fill relevant information regarding factors contributing to inconsistency in usage of family planning services. Participants were each interviewed for 20 minutes using the local language- Sesotho on the same day in order to allow them get services and proceed to their places.

\section{Data analysis}

Data was analysed using narrative and graphic form. Categorization done after data was collected, as it was easier to organize it in a visual representation and be understood.

\section{Validity and reliability}

These were ensured by doing a pilot study to ensure that the tool can be useful.

\section{Ethical considerations}

Principle of respect was maintained as the project was done after obtaining informed consent from participants and their autonomy was considered as well.

Confidentiality was ensured by not divulging their information and anonymity maintained by using codes instead of their names.

Principle of justice was maintained as they were all treated fairly as they even arrived for the services at different times.

\section{Data analysis}

Distribution of women according to reasons stated on inconsistent use

Table 1.

\begin{tabular}{|l|l|l|}
\hline Response & Frequency & Percentages \\
\hline $\begin{array}{l}\text { Those who stated } \\
\text { reasons }\end{array}$ & 15 & $75 \%$ \\
\hline $\begin{array}{l}\text { Those who were } \\
\text { not sure }\end{array}$ & 5 & $25 \%$ \\
\hline Total & 20 & $100 \%$ \\
\hline
\end{tabular}

Distribution of participants according to knowledge of benefits

Table 2.

\begin{tabular}{|l|l|l|}
\hline Responses & Frequency & Percentages \\
\hline Those who know & 10 & $50 \%$ \\
\hline Those who know few & 10 & $50 \%$ \\
\hline Total & 20 & $100 \%$ \\
\hline
\end{tabular}

Findings revealed that women still inconsistently use family planning services due to lack of access due to geography of the country, partners resistance and low level of education, about $75 \%$ of women 
had reasons though they are aware of those inconsistencies. Majority of women about $50 \%$ are aware of the benefits.

\section{Discussion of findings}

Success in preventing unintended pregnancies requires effective use of contraceptives, however this is also influenced by the method used, adherence and support by the partners as it has been observed that use of family planning services is a taboo, culturally a woman should have as many children as possible.

Women travel for long distances to get services and this contributes to inconsistent use, therefore advocacy for health posts is required to reach women at their places and counselling on the use of methods motivates women to continuously and openly use the method. Weaker adolescent corners should be revived as teenage pregnancy is on the rise with information and change of behaviour.

The importance of consistency and correct use of methods need to be emphasised as some methods are used as dual methods, that is prevention of pregnancy and sexually transmitted infections including Hiv.

Women and girls should be empowered to choose the number, timing and spacing of their pregnancies as not only a matter of health but as a human right vital to sustainable development including women's education and status in society.

\section{Conclusion}

Majority of women are aware of the benefits but they use family planning methods when convenient to them. Others are challenged by various factors such as distance from their homes to the services. Male partners are resistant to the use of family planning services as they believe that damage is done to the body and women would be promiscuous.

Also, it has been observed that there is a strong relationship between demographic factors and inconsistent use of services.

Health education is needed to be continued to all so that service use can be increased and be used consistently. Universal coverage will also decrease inconsistent use of services, as family planning is a human right.

\section{References}

[1]. Borrero s., Dehlendorf C., Rodriquez M.I., Steinauer J. Disparities in Family Planning 2006. Volume 6 Page 212.

[2]. Darroch, J.E. \&Frost, J. J. 2008. Factors Associated with Contraceptive Choice and Inconsistent Method Use, United States accessed in June 2008

[3]. Consistent and Inconsistent contraception among women 20-29. Insights from qualitative interviews working paper 1-11 May 2011.

[4]. Fairley C., Mcnamee K., Ong J., Temple-Smith M., Wong W.C.M. Contraception matters: Indicators of poor usage of contraception in sexually active women attending family planning clinics in Victoria, Australia published online 2012 December 23.

[5]. Family Planning A Global Handbook for Providers.

[6]. Finer L.B., Henshaw S.K. Disparities in rates of unintended pregnancy in United States, 1994 and 2001.

Perspective Sexual Reproductive Health2006:38:90-96 [PubMed].

[7]. Http://who.int/reproductivehealth/topics/family_planning/en/.

[8]. Http:// onlinelibrary.wiley.com/doi/10.1111/fare pdf Http://en Wikipedia.org/wiki/Family_Planning

[9]. Reproductive Journal on inconsistent fertility motivation and contraceptive use behaviours among women in Honduras. Published 09November, 2009.

[10]. Reproductive Rights affect demographic transition - UNFPA accessed from The Nation Friday 19-25 October, 2018.

[11]. www.ncbi. Nkm.nih.gov/pmc/article pmk 2835625. 